\title{
Quarkonia: Theory
}

\section{An-Ping Chen ${ }^{a}$, Yan-Qing Ma ${ }^{* b, c, d}$}

${ }^{a}$ College of Physics and Communication Electronics, Jiangxi Normal University, Nanchang 330022, China

${ }^{b}$ School of Physics and State Key Laboratory of Nuclear Physics and Technology, Peking University, Beijing 100871, China

${ }^{c}$ Center for High Energy physics, Peking University, Beijing 100871, China

${ }^{d}$ Collaborative Innovation Center of Quantum Matter, Beijing 100871, China

E-mail: chenanping@pku.edu.cn

yqma@pku.edu.cn

Recently, there have been significant progresses for theories of quarkonium production in protonproton and proton-nucleus collisions at colliders. We give a brief review of these theories, with emphasis on the successes and difficulties of nonrelativistic QCD factorization formalism, as well as the new proposal of soft gluon factorization.

HardProbes 2020

1-6 June 2020

Austin, Texas

${ }^{*}$ Speaker. 


\section{Introduction}

Heavy quarkonium production at high energy hadronic collisions is important to test our understanding of QCD. Heavy quarkonium is a bound state of a heavy quark $(Q)$ and its anti-quark $(\bar{Q})$, and a heavy quarkonium production process involves three typical momentum scales: the heavy quark mass $m_{Q}\left(m_{c} \approx 1.3 \mathrm{GeV}\right.$ and $m_{b} \approx 4.2 \mathrm{GeV}$ in $\overline{\mathrm{MS}}$ scheme), which governs the perturbative creation of the heavy quark pair $(Q \bar{Q})$; the heavy quark momentum $m_{Q} v$ in the quarkonium rest frame; and the heavy quark kinetic energy $m_{Q} v^{2}$, which governs the nonperturbative hadronization of the $Q \bar{Q}$ to physical quarkonium. Here $v$ is the typical heavy quark velocity inside of the quarkonium rest frame ( $v^{2} \approx 0.3$ for charmonium and $v^{2} \approx 0.1$ for bottomonium). Hence heavy quarkonium presents an ideal laboratory to study both perturbative and nonperturbative aspects of QCD dynamics. Moreover, in nucleus-nucleus collisions, heavy quarkonium production constitutes a sensitive probe of the hot strongly-interacting matter. Therefore, a good understanding of the heavy quarkonium production mechanism is very crucial.

\section{Quarkonium production mechanism}

Heavy quarkonium production is usually separated into two steps: (1) the production of a $Q \bar{Q}$ pair with definite spin and color state in a hard collision, which could be calculated perturbatively; and (2) hadronization of the $Q \bar{Q}$ pair into a physical heavy quarkonium at a momentum scale much less than the heavy quark mass $m_{Q}$, which is in principle a nonperturbative process. Different treatments of the nonperturbative hadronization process led to different theoretical models for quarkonium production.

In the color evaporation model (CEM) [1,2,3], it is assumed that each produced $Q \bar{Q}$ pair can evolve into a specific heavy quarkonium if its invariant mass is below the open-charm/bottom threshold. It is further assumed that the probability for the $Q \bar{Q}$ pair pair to evolve into a quarkonium state $H$ is given by a constant $F_{H}$, which is the only free parameter $F_{H}$. The CEM is intuitive and simple, but it is not very successful phenomenologically. A straightforward prediction of the CEM is that the ratio of production rate of any two different charmonium states is independent of kinematics and process. However, this prediction contradicts the data from many experiments, for example, the ratio of production cross section of $\psi(2 S)$ over that of $J / \psi$ depends on their transverse momentum [4, 5]. To overcome these obstacles, an improved version of the model, the ICEM, was proposed [6], in which kinematic effects of soft gluons emission in the hadronization process are considered. It was found that the ICEM describes the charmonium yields as well as the ratio of $\psi(2 S)$ over $J / \psi$ better than the traditional CEM.

In the color singlet model (CSM) [7, 8, 9], the $Q \bar{Q}$ pair is assumed to have the same color, spin and angular-momentum quantum numbers as the physical heavy quarkonium. Especially, it must be in a color singlet. Under this assumption, the production cross section for each quarkonium state $H$ is related to the wave-function (or its derivative) of $H$ at the origin, which can be extracted from the decay process of $H$, or be calculated from the potential model or lattice QCD. Therefore, the CSM effectively has no free parameter. The LO CSM predictions were found to underestimate the experimental data of direct $J / \psi$ and $\psi(2 S)$ production at $\sqrt{s}=1.8 \mathrm{TeV}$ [10] by more than an order of magnitude. In the past decade, it was found that, the NLO and NNLO corrections to the 
CSM are significantly larger than the LO contributions [11, 12, 13, 14]. Including these corrections can reduce the big gap between the LO CSM prediction and the data, although a full description of data is still hard. Moreover, in the case of P-wave production and decay, the CSM is known to be inconsistent because it suffers from uncanceled infrared divergences.

In the NRQCD factorization theory [15], the production cross section of a heavy quarkonium $H$ is given by the factorization formula

$$
\sigma_{H}=\sum_{n} \sigma_{n}\left(\mu_{\Lambda}\right)\left\langle\mathscr{O}_{n}^{H}\left(\mu_{\Lambda}\right)\right\rangle
$$

Here $\mu_{\Lambda}$ is the NRQCD factorization scale, which is the ultraviolet (UV) cutoff of the NRQCD effective theory, $\sigma_{n}$ is the short-distance coefficient (SDC) which describes the production of a $Q \bar{Q}$ pair with quantum number $n$ in the hard scattering, and $\left\langle\mathscr{O}_{n}^{H}\left(\mu_{\Lambda}\right)\right\rangle$ is the NRQCD long-distance matrix element (LDME) that describe the hadronization of the $Q \bar{Q}$ pair in state $n$ into the heavy quarkonium $H$. Each LDME has a definite scaling with $v$ and thus the sum over $n$ can be organized in powers of $v$. In practice, for a certain accuracy, one truncates and keeps only a few LDMEs for each $H$ production. The predictive power of the NRQCD factorization approach is relied on the validity of such a truncation, which requires a good convergence of the velocity expansion, as well as the universality of LDMEs. As shown in Eq. (2.1), the NRQCD factorization contains both color-singlet (CS) channel and color-octet (CO) channel contributions. If one set the CO contributions to be zero, one would recover the CSM. Including the CO contributions, NRQCD solved the infrared divergence problem encountered in the CSM [16]. Based on the NRQCD factorization formula, a phenomenological study requires perturbative calculations of the SDCs and nonperturbative determinations of the LDMEs. In recent years, complete NLO studies have been carried out in the NRQCD framework, and theoretical predictions for inclusive heavy quarkonium production are generally consistent with experimental data from the Tevatron and the LHC [17, 18, $19,20]$.

However, similar to the CSM, one problem with the NRQCD factorization formula is that it suffers from large high-order corrections at large $p_{T}$, which could make the perturbative expansion of the SDCs unstable. The reason for this is that the high-order corrections for some channels may receive huge power enhancements in terms of $p_{T}^{2} / m_{Q}^{2}$, which could be much larger than the suppression of $\alpha_{s}$ at large $p_{T}$. Besides, the large $\ln \left(p_{T}^{2} / m_{Q}^{2}\right)$-type logarithms also could make the perturbative expansion unreliable. Considered this, a new QCD factorization approach was proposed to describe the large- $p_{T}$ heavy quarkonium production [21, 22, 23, 24, 25], by including both single-part fragmentation contributions and double-parton fragmentation contributions. All large $\ln \left(p_{T}^{2} / m_{Q}^{2}\right)$-type logarithms can be resumed by solving a closed set of evolution equations of FFs, which makes the $\alpha_{s}$ expansion more convergent. By calculating fragmentation functions using NRQCD, as simply LO calculation in the QCD factorization can approximately reproduce the complicated NLO calculation in NRQCD framework [26].

Furthermore, studies in recent years have shown that there are still other notable issues in the application of NRQCD factorization formula to world data on quarkonium production [27, 28, 29, $30,31,32,33,34,35]$, which may be caused by the bad convergence of velocity expansion [36]. To overcome this problem, a new factorization method called soft gluon factorization (SGF) was proposed [37]. In this method, the differential cross section of the quarkonium $H$ production is 
factorized as

$$
(2 \pi)^{3} 2 P_{H}^{0} \frac{d \sigma_{H}}{d^{3} P_{H}} \approx \sum_{n} \int \frac{d^{4} P}{(2 \pi)^{4}} \mathscr{H}_{n}(P) F_{n \rightarrow H}\left(P, P_{H}\right)
$$

where $P$ is the momentum of the intermediate $Q \bar{Q}$ pair, $P_{H}$ is the momentum of $H, F_{n \rightarrow H}\left(P, P_{H}\right)$ are soft gluon distribution functions (SGDs), which describe the hadronization of the $Q \bar{Q}$ pair into physical quarkonium $H$ by emitting soft gluons. SGDs are defined by QCD fields in small loop momentum region. With an explicit definition of the small region, the SGF is demonstrate to be equivalent to the NRQCD factorization [38]. Moreover, it was shown that, by using equations of motion, one can deduce SGF from NRQCD effective field theory. Comparing with the NRQCD factorization, the advantage of SGF is that it resums the series of relativistic corrections originated from kinematic effects to all powers, which gives rise to a better convergence in velocity expansion.

\section{Quarkonium production with small $x$ effects}

The observed quarkonium suppression in nucleus-nucleus collisions was considered as a key signature of the formation of QGP. However, there are other effects that are not necessarily related to the QGP formation can lead to quarkonium suppression. Some of these effects also present in $p A$ collisions and are known as cold nuclear matter (CNM) effects. Study of $p A$ collisions can not only understand CNM effects themselves, but also help to clearly extract hot medium effects. There are several CNM effects in $p A$ collisions, the modification of the parton distribution functions in the nuclei, the parton saturation effects, the multiple scattering of partons in the nucleus before or after the hard scattering, the absorption or breakup of quarkonium states, and so on. Various approaches have been introduced to study these effects [43]. Here we emphasize two of them.

In the collinear factorization approach, cross sections of quarkonium production are assumed to be dominated by leading-twist, but using nuclear parton distribution functions (nPDFs) of the target nucleus, which are usually assumed to be different from normal PDFs by a nuclear modification factor $R_{i}^{A}\left(x, Q^{2}\right)$. The modification factor is determined by performing global fit analyses of lepton-nucleus and proton-nucleus data. It was found that there is nuclear shadowing effect, namely a suppression $\left(R_{i}^{A}\left(x, Q^{2}\right)<1\right)$ at small $x \lesssim 10^{-2}$. Nuclear shadowing leads to a corresponding depletion of quarkonium production in $p A$ with respect to $p p$ collisions [44].

In the low $p_{T}$ region, higher twist contributions as well as large $\ln (1 / x)$ contributions arise that are not accounted for in collinear factorization framework. These contributions can be systematically computed in the Color Glass Condensate (CGC) effective field theory [39, 40]. Quarkonium production can then be studied by combining the CGC with quarkonium production theories. E.g., by combining CGC with NRQCD [41, 42], good description of low $p_{T}$ quarkonium production in $p p$ and $p A$ collisions is obtained [42,45]. Moreover, results in this framework can be matched smoothly to the results obtained in the NLO NRQCD factorization framework [18, 29, 30] at high $p_{T}$, providing a unified description for quarkonium production in full $p_{T}$ region.

\section{Summary}

The traditional NRQCD factorization is quite successful in describing the quarkonium production in $p p$ collisions and $p A$ collisions. Especially, by combining NRQCD factorization for 
quarkonium with appropriate factorization approach for colliding particles, CGC effective field theory, unified description for quarkonium production in full $p_{T}$ region was obtained. However, there are some notable difficulties with the NRQCD factorization, including the polarization puzzle and the universality problem, which may be caused by the bad convergence of $v$ expansion. Hopefully, these difficulties may be resolved or relieved in the SGF framework with well controlled relativistic corrections.

\section{References}

[1] H. Fritzsch, Producing Heavy Quark Flavors in Hadronic Collisions: A Test of Quantum Chromodynamics, Phys.Lett. B67 (1977) 217 [InSP IRE].

[2] M. Gluck, J. Owens, and E. Reya, Gluon Contribution to Hadronic J/psi Production, Phys. Rev. D 17 (1978) 2324 [InSPIRE].

[3] V. D. Barger, W.-Y. Keung, and R. Phillips, On psi and Upsilon Production via Gluons, Phys. Lett. B 91 (1980) 253-258 [InSP IRE].

[4] PHENIX, A. Adare et al., Ground and excited charmonium state production in $p+p$ collisions at $\sqrt{s}=200 \mathrm{GeV}$, Phys. Rev. D85 (2012) 092004 [arXiv:1105.1966] [InSPIRE].

[5] LHCb Collaboration, R. Aaij et al., Measurement of $\psi(2 S)$ meson production in pp collisions at $\operatorname{sqrt}(s)=7 \mathrm{TeV}$, Eur.Phys.J. C72 (2012) 2100 [arXiv:1204.1258] [InSPIRE].

[6] Y.-Q. Ma and R. Vogt, Quarkonium Production in an Improved Color Evaporation Model, Phys. Rev. D94 (2016) 114029 [arXiv:1609.06042] [InSPIRE].

[7] S. Ellis, M. B. Einhorn, and C. Quigg, Comment on Hadronic Production of Psions, Phys.Rev.Lett. 36 (1976) 1263 [InSPIRE].

[8] C. Carlson and R. Suaya, Hadronic Production of $\psi / J$ Mesons, Phys.Rev. D14 (1976) 3115 [InSPIRE].

[9] C.-H. Chang, Hadronic Production of J/ $\psi$ Associated With a Gluon, Nucl.Phys. B172 (1980) 425-434 [InSPIRE].

[10] CDF Collaboration, F. Abe et al., $J / \psi$ and $\psi(2 S)$ production in p $\bar{p}$ collisions at $\sqrt{s}=1.8 \mathrm{TeV}$, Phys.Rev.Lett. 79 (1997) 572-577 [InSP IRE].

[11] P. Artoisenet, J. Lansberg, and F. Maltoni, Hadroproduction of $J / \psi$ and $\Upsilon$ in association with a heavy-quark pair, Phys.Lett. B653 (2007) 60-66 [hep-ph / 0703129 ] [InSP IRE].

[12] J. M. Campbell, F. Maltoni, and F. Tramontano, $Q C D$ corrections to $J / \psi$ and $\Upsilon$ production at hadron colliders, Phys.Rev.Lett. 98 (2007) 252002 [hep-ph/ 0703113 ] [InSP IRE].

[13] P. Artoisenet, J. M. Campbell, J. Lansberg, F. Maltoni, and F. Tramontano, $\Upsilon$ Production at Fermilab Tevatron and LHC Energies, Phys.Rev.Lett. 101 (2008) 152001 [arXiv: 0806 . 3282] [InSP IRE].

[14] H.-S. Shao, Boosting perturbative QCD stability in quarkonium production, JHEP 01 (2019) 112 [arXiv:1809.02369] [InSPIRE].

[15] G. T. Bodwin, E. Braaten, and G. P. Lepage, Rigorous QCD analysis of inclusive annihilation and production of heavy quarkonium, Phys. Rev. D51 (1995) 1125-1171 [hep-ph/ 9407339$]$ [InSPIRE]. [Erratum: Phys. Rev.D55,5853(1997)]. 
[16] G. T. Bodwin, E. Braaten, T. C. Yuan, and G. P. Lepage, $P$ wave charmonium production in B meson decays, Phys.Rev. D46 (1992) 3703-3707 [hep-ph/9208254] [InSP IRE].

[17] M. Butenschoen and B. A. Kniehl, Reconciling J/ $\psi$ production at HERA, RHIC, Tevatron, and LHC with NRQCD factorization at next-to-leading order, Phys.Rev.Lett. 106 (2011) 022003 [arXiv:1009.5662] [InSPIRE].

[18] Y.-Q. Ma, K. Wang, and K.-T. Chao, $J / \psi\left(\psi^{\prime}\right)$ production at the Tevatron and LHC at $\mathscr{O}\left(\alpha_{s}^{4} v^{4}\right)$ in nonrelativistic QCD, Phys.Rev.Lett. 106 (2011) 042002 [arXiv: 1009.3655 ] [InSP IRE].

[19] K. Wang, Y.-Q. Ma, and K.-T. Chao, $\Upsilon(1 S)$ prompt production at the Tevatron and LHC in nonrelativistic QCD, Phys.Rev. D85 (2012) 114003 [arXiv:1202.6012] [InSP IRE].

[20] B. Gong, L.-P. Wan, J.-X. Wang, and H.-F. Zhang, Complete next-to-leading-order study on the yield and polarization of Upsilon(1S,2S,3S) at the Tevatron and LHC, Phys.Rev.Lett. 112 (2014) 032001 [arXiv:1305.0748] [InSPIRE].

[21] Z.-B. Kang, Y.-Q. Ma, J.-W. Qiu, and G. Sterman, Heavy quarkonium production at collider energies: Factorization and Evolution, Phys.Rev. D90 (2014) 034006 [arXiv: 1401.0923 ] [InSP IRE].

[22] Z.-B. Kang, Y.-Q. Ma, J.-W. Qiu, and G. Sterman, Heavy Quarkonium Production at Collider Energies: Partonic Cross Section and Polarization, Phys.Rev. D91 (2015) 014030 [arXiv:1411.2456] [InSPIRE].

[23] Z.-B. Kang, J.-W. Qiu, and G. Sterman, Heavy quarkonium production and polarization, Phys.Rev.Lett. 108 (2012) 102002 [arXiv:1109.1520] [InSP IRE].

[24] Z.-B. Kang, J.-W. Qiu, and G. Sterman, Factorization and quarkonium production, Nucl.Phys.Proc.Suppl. 214 (2011) 39-43 [InSP IRE].

[25] S. Fleming, A. K. Leibovich, T. Mehen, and I. Z. Rothstein, The Systematics of Quarkonium Production at the LHC and Double Parton Fragmentation, Phys.Rev. D86 (2012) 094012 [arXiv:1207.2578] [InSPIRE].

[26] Y.-Q. Ma, J.-W. Qiu, G. Sterman, and H. Zhang, Factorized power expansion for high- $p_{T}$ heavy quarkonium production, Phys.Rev.Lett. 113 (2014) 142002 [arXiv:1407.0383] [InSP IRE].

[27] B. Gong and J.-X. Wang, QCD corrections to polarization of J/ $\psi$ and $\Upsilon$ at Tevatron and LHC, Phys.Rev. D78 (2008) 074011 [arXiv: 0805 . 2469] [InSPIRE].

[28] M. Butenschoen and B. A. Kniehl, J/ $\psi$ polarization at Tevatron and LHC: Nonrelativistic-QCD factorization at the crossroads, Phys.Rev.Lett. 108 (2012) 172002 [arXiv:1201.1872] [InSPIRE].

[29] K.-T. Chao, Y.-Q. Ma, H.-S. Shao, K. Wang, and Y.-J. Zhang, J/ $\psi$ Polarization at Hadron Colliders in Nonrelativistic QCD, Phys.Rev.Lett. 108 (2012) 242004 [arXiv: 1201.2675 ] [InSP IRE].

[30] B. Gong, L.-P. Wan, J.-X. Wang, and H.-F. Zhang, Polarization for Prompt J $\psi \psi, \psi(2 S)$ production at the Tevatron and LHC, Phys.Rev.Lett. 110 (2013) 042002 [arXiv: 1205.6682 ] [InSP IRE].

[31] G. T. Bodwin, H. S. Chung, U.-R. Kim, and J. Lee, Fragmentation contributions to J/ $\psi$ production at the Tevatron and the LHC, Phys.Rev.Lett. 113 (2014) 022001 [arXiv: 1403.3612 ] [InSP IRE].

[32] P. Faccioli, V. Knunz, C. Lourenco, J. Seixas, and H. K. Wohri, Quarkonium production in the LHC era: a polarized perspective, Phys.Lett. B736 (2014) 98-109 [arXiv: 1403 . 3970] [InSP IRE].

[33] H. Han, Y.-Q. Ma, C. Meng, H.-S. Shao, Y.-J. Zhang, and K.-T. Chao, $\Upsilon(n S)$ and $\chi_{b}(n P)$ production at hadron colliders in nonrelativistic QCD, Phys. Rev. D94 (2016) 014028 [arXiv: 1410.8537 ] [InSPIRE]. 
[34] H. S. Shao, H. Han, Y. Q. Ma, C. Meng, Y. J. Zhang, and K. T. Chao, Yields and polarizations of prompt $J / \psi$ and $\psi(2 S)$ production in hadronic collisions, JHEP 05 (2015) 103 [arXiv:1411.3300] [InSPIRE].

[35] Y.-J. Zhang, Y.-Q. Ma, K. Wang, and K.-T. Chao, QCD radiative correction to color-octet $J / \psi$ inclusive production at B Factories, Phys.Rev. D81 (2010) 034015 [arXiv:0911.2166] [InSPIRE].

[36] M. L. Mangano and A. Petrelli, NLO quarkonium production in hadronic collisions, Int. J. Mod. Phys. A12 (1997) 3887-3897 [hep-ph/9610364] [InSP IRE].

[37] Y.-Q. Ma and K.-T. Chao, New factorization theory for heavy quarkonium production and decay, Phys. Rev. D100 (2019) 094007 [arXiv: 1703 . 08402] [InSPIRE].

[38] A.-P. Chen and Y.-Q. Ma, Theory for quarkonium: from NRQCD factorization to soft gluon factorization, [arXiv:2005.08786] [InSPIRE].

[39] E. Iancu and R. Venugopalan, The Color glass condensate and high-energy scattering in QCD, [hep-ph/0303204] [InSPIRE].

[40] F. Gelis, E. Iancu, J. Jalilian-Marian, and R. Venugopalan, The Color Glass Condensate, Ann.Rev.Nucl.Part.Sci. 60 (2010) 463-489 [arXiv: 1002 .0333] [InSP IRE].

[41] Z.-B. Kang, Y.-Q. Ma, and R. Venugopalan, Quarkonium production in high energy proton-nucleus collisions: CGC meets NRQCD, JHEP 1401 (2014) 056 [arXiv:1309.7337] [InSP IRE].

[42] Y.-Q. Ma and R. Venugopalan, Comprehensive Description of J/ $\psi$ Production in Proton-Proton Collisions at Collider Energies, Phys.Rev.Lett. 113 (2014) 192301 [arXiv:1408.4075] [InSPIRE].

[43] A. Andronic et al., Heavy-flavour and quarkonium production in the LHC era: from proton-proton to heavy-ion collisions, Eur. Phys. J. C 76 (2016) 107 [arXiv: 1506.03981 ] [InSP IRE].

[44] I. Helenius, K. J. Eskola, H. Honkanen, and C. A. Salgado, Impact-Parameter Dependent Nuclear Parton Distribution Functions: EPSO9s and EKS98s and Their Applications in Nuclear Hard Processes, JHEP 07 (2012) 073 [arXiv: 1205 . 5359] [InSP IRE].

[45] Y.-Q. Ma, R. Venugopalan, and H.-F. Zhang, J/ $\psi$ production and suppression in high energy proton-nucleus collisions, Phys. Rev. D92 (2015) 071901 [arXiv: 1503.07772 ] [InSP IRE]. 